OPEN ACCESS

Edited by:

Shengjun Wang,

Jiangsu University Affiliated People's

Hospital, China

Reviewed by:

Gabriela Hernandez-Molina, Instituto Nacional de Ciencias Médicas y Nutrición Salvador Zubirán (INCMNSZ), Mexico

Federica Pedica,

San Raffaele Scientific Institute (IRCCS), Italy

${ }^{*}$ Correspondence: Jingyang $L$

wgzncsu@csu.edu.cn

Specialty section: This article was submitted to Autoimmune and Autoinflammatory Disorders, a section of the journal

Frontiers in Immunology

Received: 31 May 2021

Accepted: 26 July 2021

Published: 10 August 2021

Citation:

Wang G, Zhuo N, Luo X, Tian F, Wen Z and Li J (2021) IgG4-Related Disease

With Testicular Involvement: A Case

Report and Review of Literature.

Front. Immunol. 12:717902

doi: 10.3389/fimmu.2021.717902

\section{IgG4-Related Disease With Testicular Involvement: A Case Report and Review of Literature}

\author{
Gang Wang ${ }^{1}$, Ning Zhuo ${ }^{2}$, Xiaowen Luo ${ }^{1}$, Feng Tian ${ }^{1}$, Zhenhua Wen ${ }^{1}$ and Jingyang $L i^{1 *}$ \\ 1 Department of Rheumatology and Immunology, Zhuzhou Hospital Affiliated to Xiangya Medical College, Central South University, \\ Zhuzhou, China, ${ }^{2}$ Department of Nephrology, The Second Xiangya Hospital, Central South University, Changsha, China
}

Immunoglobulin G4-related disease (lgG4-RD) is an autoimmune inflammatory disease characterized by infiltration of lgG4+ plasma cells that can simulate a tumor manifesting as a tumor-like mass. This disease involves the pancreas, biliary tract, kidneys, salivary glands, lymph nodes, aorta, and retroperitoneum amongst other organs. However, testicular involvement is a rare entity in this disease. The treatment of testicular involvement in lgG4-RD is currently controversial. We present the case of a 65-year-old man with swelling and pain in his right scrotum three months ago. On examination, a mobile mass of approximately $2 \mathrm{~cm}$ in diameter was found in the right scrotum. Serological tests showed elevated levels of lgG4 and negative for tumor markers. Enhanced computed tomography of the scrotum showed a nodular hyperdense shadow with a diameter of approximately $23 \mathrm{~mm}$ on the right epididymis. Pathological biopsy of the right epididymis showed infiltration of plasma cells, lymphocytes, and a few neutrophils. IgG4+ plasma cells stained positive, with an lgG4/lgG ratio of more than $40 \%$ and more than 30 lgG4+ plasma cells per high-power field. A diagnosis of lgG4-RD involving the testicles was made. Prednisone $30 \mathrm{mg} / \mathrm{d}$ was given for three weeks. No scrotum swelling or pain was observed at the follow-up after six months. IgG4-related disease should be considered whenever a mass-like lesion with typical histomorphologic features involving multiple organs/anatomical sites is encountered. The testicles are an important male reproductive organ, especially for young male patients with fertility requirements. For patients with IgG4-RD testicular involvement, surgical or medical treatment requires further study.

Keywords: IgG4-related disease, testis, clinical feature, treatment, glucocorticoids

\section{INTRODUCTION}

IgG4-RD is an autoimmune inflammatory disease characterized by infiltration of IgG4+ plasma cells that can simulate a tumor manifesting as a tumor-like mass (1). Also, IgG4-RD is a systemic disease that involves the pancreas, biliary tract, kidneys, salivary glands, lymph nodes, aorta, and retroperitoneum amongst other organs (2). However testicular involvement is a rare entity in this disease. Until now, IgG4-RD testicular involvement has been described mainly in the form of case reports, with a lack of summary studies. Since the testis is a rarely affected entity in IgG4-RD and its 
clinical features are poorly understood, the purpose of this article is to raise awareness of the disease, as well as to present problems and phenomena that may need to be addressed in the future.

\section{CASE PRESENTATION}

The patient, a 65-year-old man, presented with swelling and pain in his right scrotum three months ago. He was first admitted to our hospital 5 years ago with a cough and fever. The CT scan of the abdomen showed multiple soft tissue densities behind the peritoneum and the serum IgG4 $3200 \mathrm{mg} / \mathrm{dL}$. The patient was definitively diagnosed with IgG4-RD as well as secondary retroperitoneal fibrosis by the gold standard of histopathology. He was in long-term remission after regular treatment with mycophenolate mofetil (1g Bid) and prednisone $(0.6 \mathrm{mg} / \mathrm{kg})$, followed by six months, four years, and a recent abdominal CT scan showed no progression of retroperitoneal fibrosis. He had no previous history of pulmonary or urinary tuberculosis and no significant family history. On examination, a mobile mass of approximately $2 \mathrm{~cm}$ in diameter was found in the right scrotum. The mass was hard and tender. No other positive signs were found. Laboratory tests showed erythrocyte sedimentation rate $69 \mathrm{~mm} / \mathrm{h}$ (reference range $0-15 \mathrm{~mm} / \mathrm{h}$ ), C-reactive protein 29.9 $\mathrm{mg} / \mathrm{L}$ (reference range $0-10 \mathrm{mg} / \mathrm{L}$ ), complement C3 $0.58 \mathrm{~g} / \mathrm{L}$ (reference range 0.9-1.8 g/L), complement C4 $0.03 \mathrm{~g} / \mathrm{L}$ (reference range 0.1-0.4 g/L). Serological examination showed elevated IgG4 levels to $2070 \mathrm{mg} / \mathrm{dL}$ (reference range $6-130 \mathrm{mg} / \mathrm{dL}$ ).
Negative for prostate-specific antigen (PSA), tumor markers, and tuberculosis antibodies. Scrotal ultrasound demonstrating enlargement of the right testicle. Enhanced computed tomography of the scrotum showed a nodular hyperdense shadow with a diameter of approximately $23 \mathrm{~mm}$ on the right epididymis, with significant heterogeneous enhancement on the enhanced scan (Figure 1). After ruling out testicular tuberculosis and hydrocele due to retroperitoneal fibrosis secondary to IgG4$\mathrm{RD}$, given the potential risk of malignancy, he underwent a pathological biopsy by puncture of the right epididymis. Pathological biopsy of the right epididymis showed infiltration of plasma cells, lymphocytes, and a few neutrophils with occlusive vasculitis changes in the tissue (Figure 2A). IgG4+ plasma cells stained positive, with an IgG4/IgG ratio of more than $40 \%$ and more than $30 \mathrm{IgG} 4+$ plasma cells per high-power field (Figure 2B). A diagnosis of IgG4-RD involving the testicles was made. Prednisone $30 \mathrm{mg} / \mathrm{d}$ was given for three weeks. No scrotum swelling or pain was observed at the follow-up after six months. The timeline of diagnosis, treatment, and prognosis for this case is shown in Figure 3.

\section{METHODS}

We performed a literature review of published articles in English on IgG4-RD involving the testis. We searched electronic databases (Pubmed, Embase, Cochrane Library) using the following keywords: "epididymis", "paratestis", "testis",

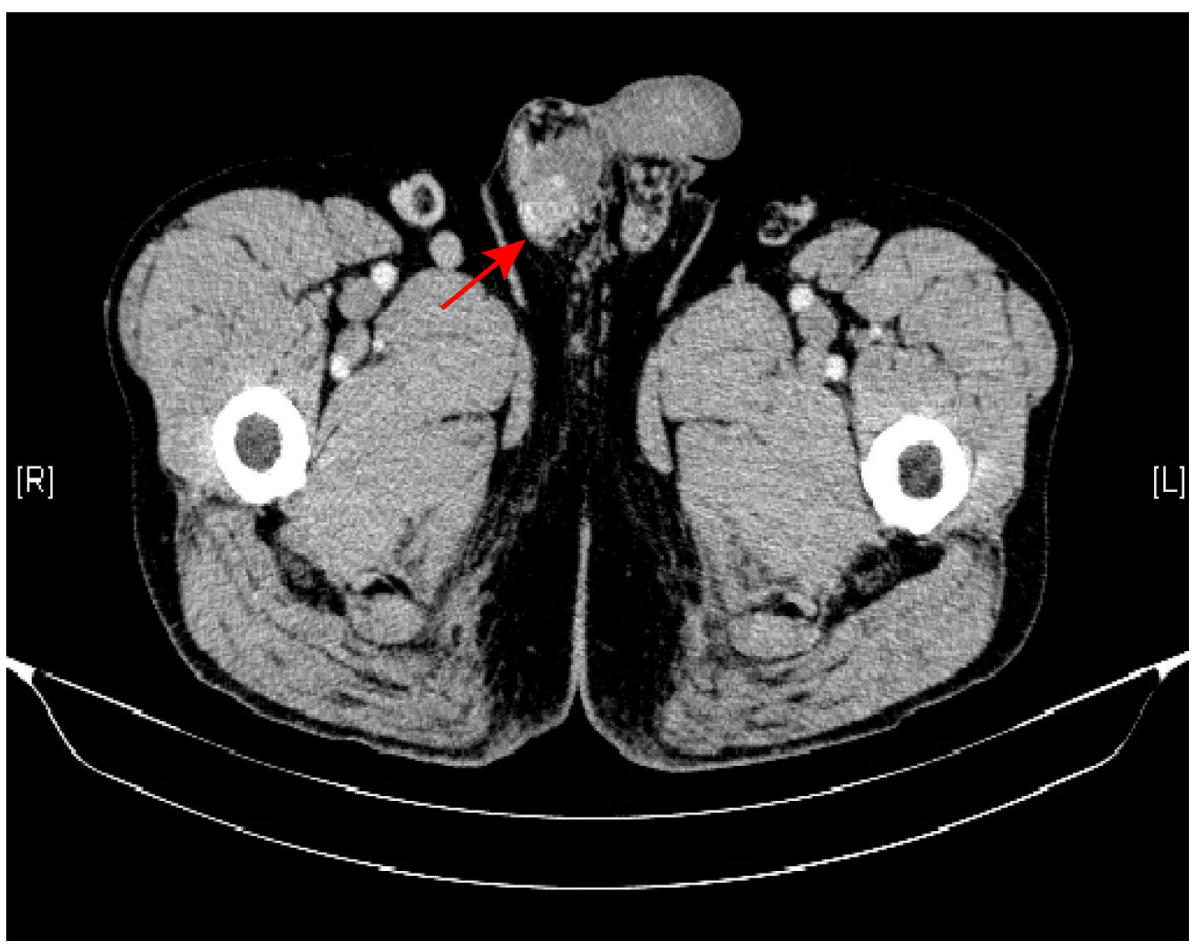

FIGURE 1 | Enhanced computed tomography of the scrotum showed a nodular hyperdense shadow in the right epididymis (arrow). 

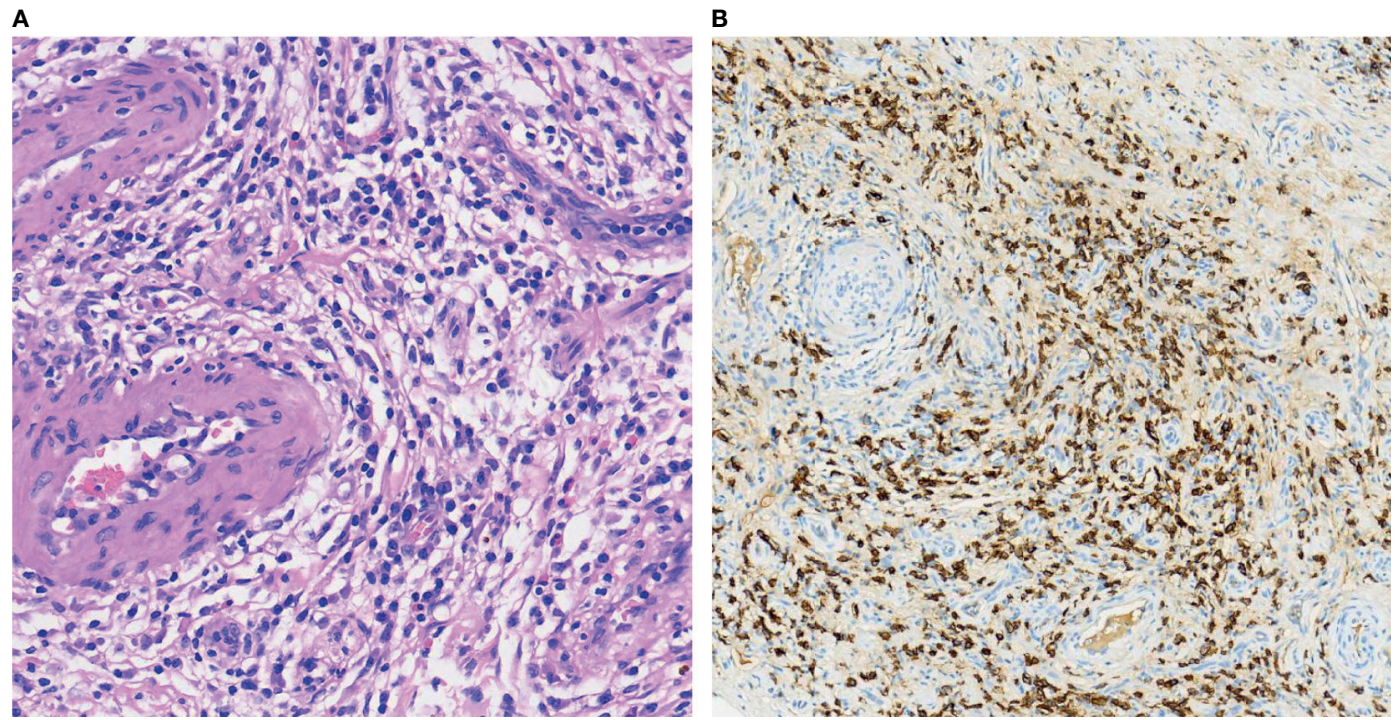

FIGURE 2 | Pathological biopsy of the right epididymis showed infiltration of plasma cells, lymphocytes, and a few neutrophils with occlusive vascultis changes in the tissue (HE, $\times 40)$ (A). Immunostains showed a markedly increased number of lgG4+ plasma cells as well as an increased lgG4+/lgG+ ratio (IgG4 immunostain, $\times 40)$ (B)

\begin{tabular}{|c|c|c|c|}
\hline $\begin{array}{l}\text { The patient came to our hospital } \\
\text { for the first time. } \\
\text { Histopathological examination } \\
\text { revealed IgG-RD with secondary } \\
\text { retroperitoneal fibrosis. }\end{array}$ & \multicolumn{2}{|c|}{$\begin{array}{l}\text { A CT scan of the abdomen showed } \\
\text { that the density of multiple soft } \\
\text { tissue in the retroperitoneum was } \\
\text { significantly reduced. There were } \\
\text { no adverse drug reactions. }\end{array}$} & $\begin{array}{l}\text { Follow-up after } 6 \text { months } \\
\text { showed that the patient } \\
\text { recovered well, with no } \\
\text { recurrence of scrotal } \\
\text { swelling or pain. }\end{array}$ \\
\hline \multicolumn{2}{|l|}{$2016 / 6 / 17$} & \multicolumn{2}{|c|}{$2020 / 11 / 27$} \\
\hline \begin{tabular}{l|l}
$2016 / 5 / 18$ & \\
\end{tabular} & & $2021 / 5 / 1$ \\
\hline \multicolumn{2}{|c|}{$\begin{array}{l}\text { The patient was treated with } \\
\text { mycophenolate mofetil } 1 \mathrm{~g} \mathrm{Bid} \\
\text { and prednisone } 0.6 \mathrm{mg} / \mathrm{kg} \text {, } \\
\text { followed by long-term remission. }\end{array}$} & \multicolumn{2}{|c|}{$\begin{array}{l}\text { The patient presented with right scrotal } \\
\text { swelling and pain for } 3 \text { months. Testicular } \\
\text { biopsy revealed that } \mathrm{IgG} 4-\mathrm{RD} \text {, and treatment } \\
\text { with prednisone } 0.6 \mathrm{mg} / \mathrm{kg} \text { was initiated. }\end{array}$} \\
\hline
\end{tabular}

FIGURE 3 | Timeline of diagnosis, treatment, and prognosis in this case.

“testicular”, "scrotum”, “scrotal”, "intrascrotal”, "spermatic cord, "paratesticular fibrous pseudotumor", "paratesticular pseudotumor", "Epididymo-orchitis", "Immunoglobulin G4", "IgG4". It is worth noting that the literature was searched by two researchers, and a third researcher made the final judgment when there was disagreement. We also reviewed the articles cited in the literature to ensure the accuracy of the retrieved content. Finally, 14 articles were included in the study (Table 1).

\section{DISCUSSION}

IgG4-RD is a chronic progressive autoimmune disease characterized by elevated serum IgG4 levels as well as IgG4+ plasma cell infiltration of organs (1). The pathogenesis of IgG4-RD is still unclear, but the main view is that autoimmunity and infection are potential immune triggers for IgG4-RD, leading to overexpression of cytokines represented by type 2 helper T (Th2) cells and activation of regulatory $\mathrm{T}$ (Treg) cells causing massive infiltration of inflammatory cells leading to organ damage (16). IgG4-RD can involve multiple organs throughout the body, and the testis, a rare area of involvement, may also present with mass forming lesions.

A total of 18 patients with a median age of 46 years and a mean age of 47.5 years were included in the study. There is a bimodal age distribution with a concentration of people aged 2050 years and 70 years or older. A cohort study by Inoue et al. (17) enrolled 235 IgG4-RD patients with a median age of 67 years. 
TABLE 1 | Clinical characteristics of 18 patients with testicular involvement of IgG4-related diseases.

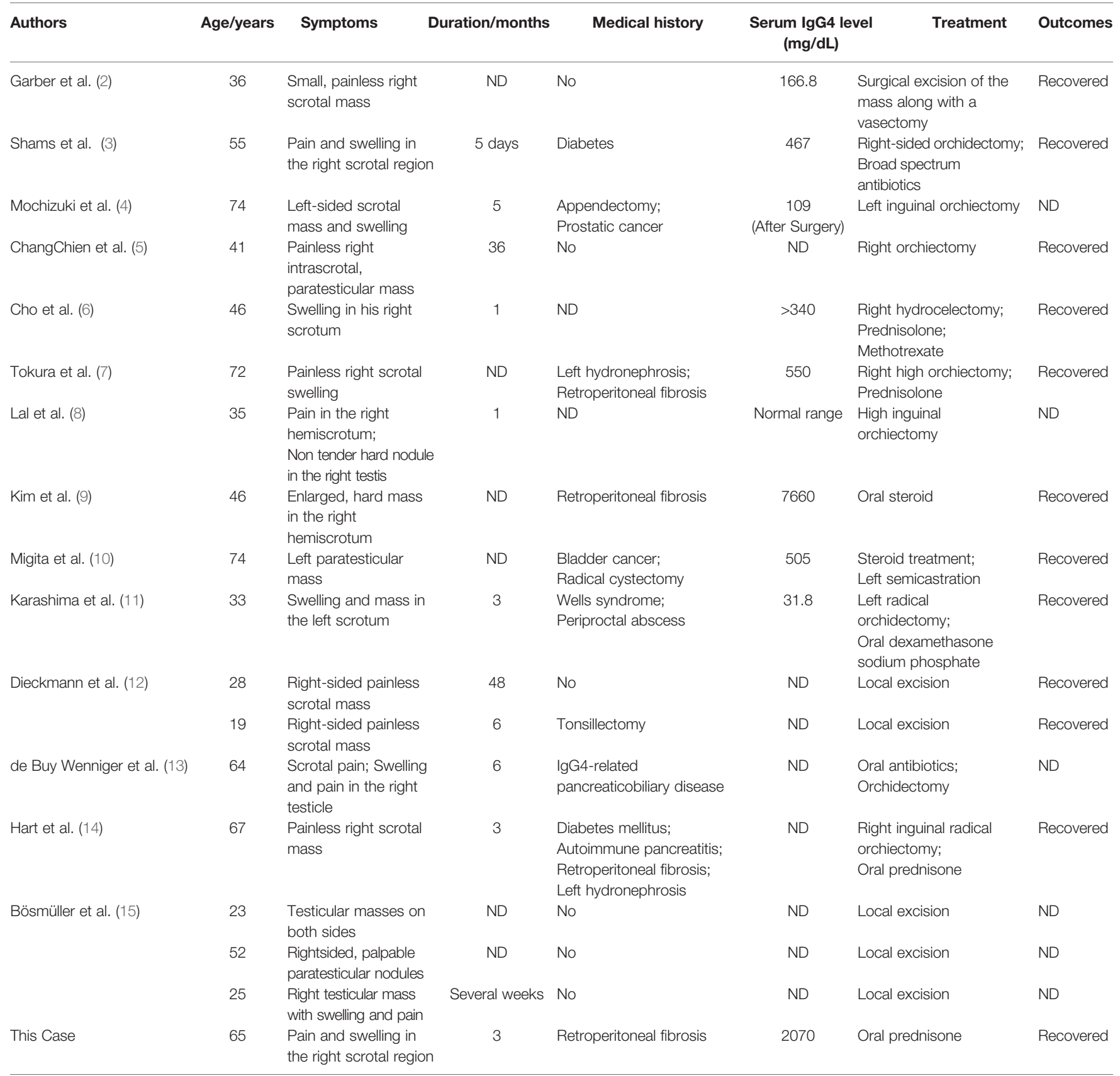

ND, Not described.

Wallace et al. (18) enrolled 125 IgG4-RD patients with a mean age of 50.3 years. The above data indicate that the mean age of patients with testicular involvement is much younger. A prospective study that included 737 patients with IgG4-RD showed that the proportion of superficial organ (such as the testicles) involvement decreased with age, while the proportion of visceral organ involvement increased with age, which was more visible in male patients (19). However, the patients included in this article did not fit the above findings. In terms of clinical manifestations, intra-scrotal masses were the most frequent $(13 / 18,72.22 \%)$, followed by swelling $(8 / 18,44.44 \%)$ and pain $(5 / 18,27.77 \%)$. Testicular painlessness was more common than patients with pain (13 vs 5). Testicular painlessness may be the cause of delayed diagnosis and the prolonged course of the disease. Six patients (33.33\%) had no previous underlying disease and subsequently developed IgG4$\mathrm{RD}$, while four patients (22.22\%) developed retroperitoneal fibrosis before the IgG4-RD involvement of the testicles. The relationship between retroperitoneal fibrosis and testicular involvement is not clarified. We hypothesize that when retroperitoneal fibrosis developed, the patient's immune system had been attacked and no longer provided complete protection 
to the testicles. In terms of treatment, 16 patients $(88.88 \%)$ underwent surgical treatment including partial and radical resection; only 7 patients were treated with hormone therapy $(38.88 \%)$ and 1 patient was treated with methotrexate. Twelve patients were followed up and all improved.

Swelling and pain associated with IgG4-RD in the testis should be differentiated from testicular tuberculosis, testicular tumors, and hydrocele due to retroperitoneal fibrosis secondary to IgG4-RD. The patient had no previous history of tuberculosis of the lung or urinary tract, which effectively ruled out testicular tuberculosis. Secondary retroperitoneal fibrosis of IgG4-RD involving the ureter, inferior vena cava, abdominal aorta, and its branches, leading to hydrocele testis, can be the cause of testicular enlargement. However, after the patient was regularly treated with prednisone and mycophenolate mofetil for the first time in our hospital, retroperitoneal fibrosis was long in remission, which was confirmed by multiple abdominal CTs. Histopathologic diagnosis is still the gold standard for the diagnosis of this disease. Therefore, the patient ruled out any of these causes. The fact that this disease can mimic testicular malignancy and the inexperience of surgeons with this disease lead the vast majority of patients to receive surgical treatment. For the treatment of definitively diagnosed
IgG4-RD, steroid hormone therapy that includes both inductions of remission and maintenance therapy may be more beneficial in rapidly resolving the patient's symptomatic, biochemical, and imaging abnormalities (20). The testicles are a vital reproductive organ that plays an important role in regulating growth and development and maintaining male secondary sexual characteristics. A thorough evaluation should be performed especially in young patients with fertility requirements. It is necessary to enlist the assistance of rheumatologists and pathologists in cases of diagnostic difficulties. Early diagnosis and management can prevent unnecessary orchiectomy.

Glucocorticoids are the first-line agents to induce remission in patients with IgG4-RD (20). Patients with poor response to glucocorticoids should undergo additional evaluation, including repeat biopsy procedures to verify the diagnosis (20). Immunosuppressants can be an alternative option to poor hormone efficacy. Prevention of IgG4-RD relapse is the focus of maintenance therapy. In general, the risk of relapse is highest when patients present with multiple organ involvement, elevated serum IgG4, IgE, and peripheral eosinophilia (1). Thus, the importance of long-term monitoring of patient's serum IgG4, $\operatorname{IgE}$, and peripheral eosinophils is highlighted.

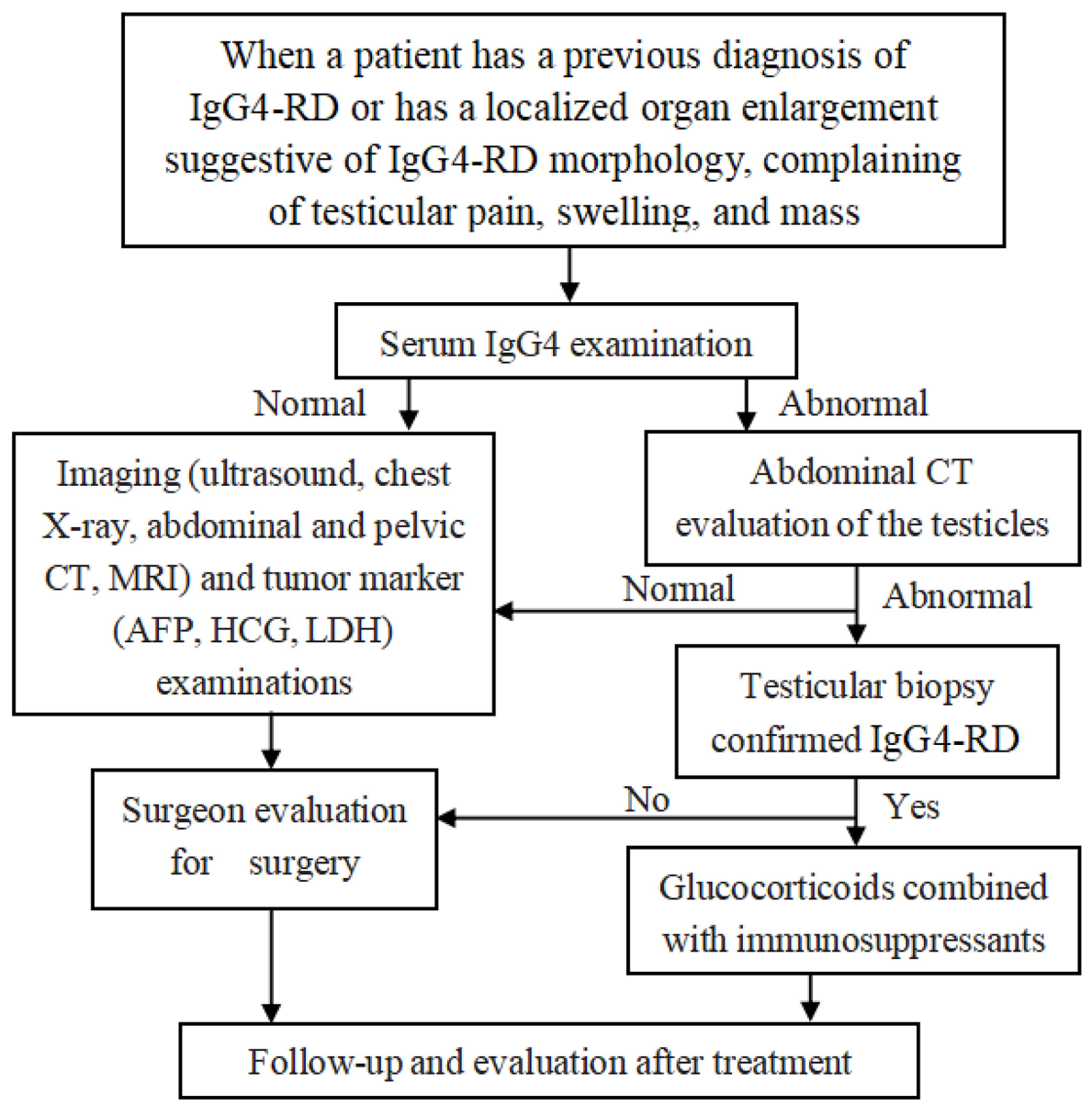

FIGURE 4 | The empirically tailored, step-wise approach to the current treatment of testicular pain, swelling, and mass. 
In clinical practice, in addition to careful physical examination, when a patient has a previous diagnosis of IgG4-RD or has a localized organ enlargement suggestive of IgG4-RD morphology, complaining of testicular pain, swelling, and mass, testing the serum IgG4 level is essential as it plays a vital role in the diagnosis and monitoring of IgG4-RD recurrence (6). When serum IgG4 is normal, the surgeon's evaluation is a priority and important. The screening of imaging and tumor markers can rule out testicular tumors as early as possible. However, testicular computed tomography and testicular aspiration biopsy are preferred and important when serum IgG4 is abnormal. We recommend the involvement of rheumatologists and pathologists throughout the entire process for the early diagnosis of IgG4-RD. The disease is responsive to glucocorticoid therapy, and early diagnosis and management can prevent unnecessary orchiectomy. Notably, regular follow-up after treatment allows early detection of disease evolution (Figure 4). The patient also presented his point of view: when there is testicular swelling or pain, timely medical attention is crucial to avoid delay in the illness.

This study has some limitations. Testicular involvement is uncommon, thus besides presenting the case, it is difficult to provide definitive conclusions. At the same time, the data for this study is based on published literature, which suggests that there may be more cases of testicular involvement of IgG4-RD in real life than in published literature. However, based on the existing evidence, this may provide an important reference for future research and cause for concern.

Finally, we found it interesting that the right testis was significantly more involved than the left (15 vs 4), only Bösmüller et al. (15) reported a patient with testicular involvement on both sides. However, there are no studies that describe how the right testis predominates in this disease, and we speculate that it may be related to the local physiological anatomy. Information on the testicular endocrine function such as androgen levels and sperm production is not available in the literature. Further studies are needed to confirm whether this disease affects testicular endocrine function and by what pathway.

\section{REFERENCES}

1. Mahajan VS, Mattoo H, Deshpande V, Pillai SS, Stone JH. IgG4-Related Disease. Annu Rev Pathol (2014) 9:315-47. doi: 10.1146/annurev-pathol-012513-104708

2. Garber ME, Wu A, Millet JD. IgG4-Related Disease Presenting as a Solitary Paratesticular Fibrous Pseudotumor. Urol Case Rep (2021) 36:101584. doi: 10.1016/j.eucr.2021.101584

3. Shams A, Das A, Sinha M, Gulati N, Mehndiratta MM, Kaushik M, et al. IgG4-Related Disease With Selective Testicular Involvement- A Rare Entity: Case Report With Review of Literature. Turk Patoloji Derg (2021) 37(1):7883. doi: 10.5146/tjpath.2020.01493

4. Mochizuki K, Hanai Y, Nakazawa K, Murata S, Kasai K, Mochizuki N, et al. IgG4-Related Disease of the Paratestis With the Scrotal Fluid: A Case Report. Diagn Cytopathol (2019) 47(2):130-3. doi: 10.1002/dc.24099

5. ChangChien YC, Kovács I, Hargitai Z, Magyar L. Paratesticular Fibrous Pseudotumor: A New Entity of IgG4-Related Disease? Ann Clin Lab Sci (2018) 48(3):381-5

6. Cho YJ, Jung WY, Lee SY, Song JS, Park HJ. Perirenal Capsule and Scrotal Involvement in Immunoglobulin G4-Related Kidney Disease: Case-Based Review. Rheumatol Int (2018) 38(10):1941-8. doi: 10.1007/s00296-018-4089-y

\section{CONCLUSION}

IgG4-related disease should be considered whenever a mass-like lesion with typical histomorphologic features involving multiple organs/anatomical sites is encountered. The involvement of rheumatologists and pathologists throughout the entire process is vital for the diagnosis of IgG4-RD. Early diagnosis and management can prevent unnecessary orchiectomy.

\section{DATA AVAILABILITY STATEMENT}

The original contributions presented in the study are included in the article. Further inquiries can be directed to the corresponding author.

\section{ETHICS STATEMENT}

Written informed consent was obtained from the individual(s) for the publication of any potentially identifiable images or data included in this article.

\section{AUTHOR CONTRIBUTIONS}

GW and NZ designed and wrote the paper. XL, FT, ZW, and JL reviewed and edited the manuscript. All authors contributed to the article and approved the submitted version.

\section{ACKNOWLEDGMENTS}

We would like to thank the members and staff of the Department of Rheumatology and Immunology of the Zhuzhou Central Hospital who contributed to this manuscript.

7. Tokura Y, Kobayashi M, Kusakabe T, Kamai T. Case of Hydrocele Testis Caused by Vaginalitis, a Rare Manifestation of Immunoglobulin G4-Related Disease. Int J Urol (2017) 24(10):771-3. doi: 10.1111/iju.13430

8. Lal J, Bhat S, Doddamani S, Devi L. Isolated Testicular Immunoglobulin G4Related Disease: A Mimicker of Malignancy. Indian J Urol (2016) 32(4):3268. doi: 10.4103/0970-1591.191273

9. Kim KH, Sung DJ, Han NY, Park BJ, Kim MJ, Cho SB, et al. Immunoglobulin G4-Related Paratesticular Fibrous Pseudotumor and Retroperitoneal Fibrosis: A Case Report. Urol Int (2015) 94(3):369-72. doi: 10.1159/000362215

10. Migita K, Miyashita T, Mizuno A, Jiuchi Y, Ito M, Matsuo M, et al. IgG4-Related Epididymo-Orchitis Associated With Bladder Cancer: Possible Involvement of BAFF/BAFF-R Interaction in IgG4-Related Urogenital Disease. Mod Rheumatol (2014) 24(1):188-94. doi: 10.3109/14397595.2013.852841

11. Karashima T, Taniguchi Y, Shimamoto T, Nao T, Nishikawa H, Fukata S, et al. IgG4-Related Disease of the Paratestis in a Patient With Wells Syndrome: A Case Report. Diagn Pathol (2014) 9:225. doi: 10.1186/s13000014-0225-5

12. Dieckmann KP, Struss WJ, Frey U, Nahler-Wildenhain M. Paratesticular Fibrous Pseudotumor in Young Males Presenting With Histological Features of IgG4-Related Disease: Two Case Reports. J Med Case Rep (2013) 7:225. doi: $10.1186 / 1752-1947-7-225$ 
13. de Buy Wenniger LM, Scheltema JM, Verheij J, Beuers U. Testicular Inflammation as a New Manifestation of IgG4-Associated Disease. Urology (2013) 82(2):e15-16. doi: 10.1016/j.urology.2013.04.046

14. Hart PA, Moyer AM, Yi ES, Hogan MC, Pearson RK, Chari ST. IgG4Related Paratesticular Pseudotumor in a Patient With Autoimmune Pancreatitis and Retroperitoneal Fibrosis: An Extrapancreatic Manifestation of IgG4-Related Disease. Hum Pathol (2012) 43(11):2084-7. doi: 10.1016/ j.humpath.2012.05.017

15. Bösmüller $\mathrm{H}$, von Weyhern $\mathrm{CH}$, Adam P, Alibegovic V, Mikuz G, Fend F. Paratesticular Fibrous Pseudotumor-an IgG4-Related Disorder? Virchows Arch (2011) 458(1):109-13. doi: 10.1007/s00428-010-0995-4

16. Stone JH, Zen Y, Deshpande V. IgG4-Related Disease. N Engl J Med (2012) 366(6):539-51. doi: 10.1056/NEJMra1104650

17. Inoue D, Yoshida K, Yoneda N, Ozaki K, Matsubara T, Nagai K, et al. IgG4Related Disease: Dataset of 235 Consecutive Patients. Medicine (Baltimore) (2015) 94(15):e680. doi: 10.1097/MD.0000000000000680

18. Wallace ZS, Deshpande V, Mattoo H, Mahajan VS, Kulikova M, Pillai S, et al. IgG4-Related Disease: Clinical and Laboratory Features in One Hundred Twenty-Five Patients. Arthritis Rheumatol (Hoboken NJ) (2015) 67(9):246675. doi: 10.1002/art.39205

19. Lu H, Teng F, Zhang P, Fei Y, Peng L, Zhou J, et al. Differences in Clinical Characteristics of IgG4-Related Disease Across Age Groups: A Prospective
Study of 737 Patients. Rheumatology (Oxford England) (2021) 60(6):2635-46. doi: 10.1093/rheumatology/keaa651

20. Lanzillotta M, Mancuso G, Della-Torre E. Advances in the Diagnosis and Management of IgG4 Related Disease. BMJ (Clinical Res ed) (2020) 369: m1067. doi: 10.1136/bmj.m1067

Conflict of Interest: The authors declare that the research was conducted in the absence of any commercial or financial relationships that could be construed as a potential conflict of interest.

Publisher's Note: All claims expressed in this article are solely those of the authors and do not necessarily represent those of their affiliated organizations, or those of the publisher, the editors and the reviewers. Any product that may be evaluated in this article, or claim that may be made by its manufacturer, is not guaranteed or endorsed by the publisher.

Copyright (c) 2021 Wang, Zhuo, Luo, Tian, Wen and Li. This is an open-access article distributed under the terms of the Creative Commons Attribution License (CC BY). The use, distribution or reproduction in other forums is permitted, provided the original author(s) and the copyright owner(s) are credited and that the original publication in this journal is cited, in accordance with accepted academic practice. No use, distribution or reproduction is permitted which does not comply with these terms. 